\title{
Lipopolysaccharide-activated bone marrow-derived dendritic cells suppress ovalbumin(OVA)-induced asthma by ameliorating the immune microenvironment
}

\author{
Zhihui Min ${ }^{1}$, Yuzhen Zeng ${ }^{1}$, Tao Zhu ${ }^{2}$, Bo Cui ${ }^{1}$, Ruolin $\mathrm{Mao}^{1}$, Meiling $\mathrm{Jin}^{1}$, and Zhihong \\ Chen $^{1}$ \\ ${ }^{1}$ Zhongshan Hospital Fudan University \\ ${ }^{2}$ Second Affiliated Hospital of Chongqing Medical University
}

July 20, 2020

\begin{abstract}
LPS produced by Gram-negative bacteria effectively stimulates the maturation of BMDCs. Previous studies have shown that DClps might induce tolerance in autoimmune diseases and cancer in vivo, whereas it remains unclear whether DClps can modulate the immune microenvironment in allergic asthma. We sought to elucidate the potential effects of DClps on OVAsensitized/challenged airway inflammation in a mouse model of asthma, which may help facilitate the application of specific tolDCs in allergic asthma patients in the future. We generated and obtained DClps from wild-type mice to evaluate their functional characteristics by ELISA and FACS. We also induced OVA-sensitized/challenged asthmatic mice and intraperitoneally treated these mice with DClps to assess the effects of these injected cells by histopathologic analysis and performing inflammatory cell counts in BALF. Changes in memory CD4 $\mathrm{T}$ cells, Tregs and phosphorylated protein in lung digests were analyzed. DClps exhibited lower levels of CD80 and MHCII and increased levels of anti-inflammatory cytokines such as IL-10 and TGF- $\beta$ than DCia. Additionally, DClps treatment dramatically ameliorated airway inflammation and diminished the infiltration of pulmonary inflammatory cells. In addition, we prolonged the modeling time of asthmatic mice and demonstrated that DClps treatment decreased the proliferation activity of pulmonary memory CD4 $\mathrm{T}$ cells, which further rendered the downregulation of Th2 cytokines. However, the number of pulmonary Tregs did not discernibly change. DClps treatment also markedly reduced the phosphorylation level of STAT6 protein.
\end{abstract}

Lipopolysaccharide-activated bone marrow-derived dendritic cells suppress ovalbumin(OVA)induced asthma by ameliorating the immune microenvironment

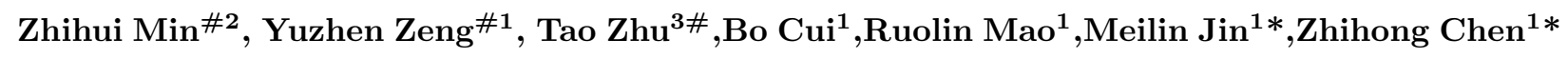

1. Respiratory Division of Zhongshan Hospital, Shanghai Institute of Respiratory Disease, Fudan University, Shanghai, China

2. Research Center of Zhongshan Hospital, Fudan University, Shanghai, China

3. Department of Respiratory Medicine, Second Affiliated Hospital of Chongqing Medical University, Chongqing, China

*Correspondence:

Zhihong Chen, MD, PhD. Respiratory Division of Zhongshan Hospital, Shanghai Institute of Respiratory Diseases, Fudan University, No. 180 Fenglin Road, Shanghai, China. Tel: 86-021-64041990-2445. Fax: 86-021-64187165. E-mail: czh60@hotmail.com 
Meilin Jin MD, PhD. Respiratory Division of Zhongshan Hospital, Shanghai Institute of Respiratory Diseases, Fudan University, No. 180 Fenglin Road, Shanghai, China. E-mail: jin.meilin@zs-hospital.sh.cn

\# Zhihui Min, Yuzhen Zeng, Tao Zhu contributed equally to this paper.

\section{Abstract}

Background: Lipopolysaccharide (LPS) produced by Gram-negative bacteria effectively stimulates the maturation of bone marrow (BM)-derived dendritic cells (BMDCs). Previous studies have shown that LPSactivated BMDCs (DClps) might induce tolerance in autoimmune diseases and cancer in vivo, whereas it remains unclear whether DClps can modulate the immune microenvironment in allergic asthma.

Objectives: We sought to elucidate the potential effects of DClps on OVA-sensitized/challenged airway inflammation in a mouse model of asthma, which may help facilitate the application of specific tolerogenic dendritic cells (tolDCs) in allergic asthma patients in the future.

Methods: We generated and obtained DClps from wild-type mice to evaluate their functional characteristics by ELISA and FACS. We also induced OVA-sensitized/challenged asthmatic mice and intraperitoneally treated these mice with DClps to assess the effects of these injected cells by histopathologic analysis and performing inflammatory cell counts in bronchoalveolar lavage fluid (BALF). Changes in memory CD4+ T cells, regulatory $\mathrm{T}$ cells (Tregs) and phosphorylated protein in lung digests were analyzed.

Results: DClps exhibited lower levels of CD80 and MHC class II molecules (MHCII) and increased levels of anti-inflammatory cytokines such as IL-10 and TGF- $\beta$ than immature DCs (DCia). Additionally, DClps treatment dramatically ameliorated airway inflammation and diminished the infiltration of pulmonary inflammatory cells. In addition, we prolonged the modeling time of asthmatic mice and demonstrated that DClps treatment decreased the proliferation activity of pulmonary memory CD4 $+\mathrm{T}$ cells, which further rendered the downregulation of type $2 \mathrm{~T}$ helper (Th2) cytokines. However, the number of pulmonary Tregs did not discernibly change. DClps treatment also markedly reduced the phosphorylation level of STAT6 protein.

Conclusion: Our findings demonstrate that LPS stimulation may lead to a tolerogenic phenotype in BMDCs, which can induce tolerance and regulate the microenvironment of asthmatic mice, possibly through the upregulation of anti-inflammatory cytokines, inhibition of pulmonary memory CD4+ T cells and downregulation of STAT6 phosphorylation.

\section{Keywords}

asthma; lipopolysaccharide; bone marrow-derived dendritic cells; memory CD4+ T cell

\section{Abbreviations}

BMDCs, bone marrow-derived dendritic cells; DClps, LPS-activated BMDCs; tolDCs, tolerogenic dendritic cells; Tregs, regulatory T cells; DCia, immature DCs; DC10, IL-10-induced DCs; $\mathrm{T}_{\mathrm{CM}}$ cells, central memory $\mathrm{T}$ cells; $\mathrm{T}_{\mathrm{EM}}$ cells, effector memory $\mathrm{T}$ cells.

DCs are considered essential for regulating CD4 T cell immunity, controlling Th1 and Th2 differentiation, inducing Tregs, and mediating tolerance ${ }^{1}$. Physiologically, immature DCs are distributed in peripheral tissues; upon stimulation by proinflammatory factors (LPS or CD40 ligand), immature DCs can further differentiate into mature DCs that express high levels of CD80 and CD86 and migrate to secondary lymphoid organs to elicit adaptive immune responses ${ }^{2}$. Nevertheless, DCs may present distinct phenotypic and functional characteristics according to the stimulus that they received. Ample evidence has demonstrated that DCs differentiated under certain conditions are capable of inducing immune tolerance in multiple disease models ranging from autoimmune to allergic diseases ${ }^{3-7}$. These specific tolDCs are distinguished by the decreased expression of costimulatory molecules and proinflammatory cytokines along with enhancement of anti-inflammatory cytokines, thus leading to T cell anergy and Treg activation ${ }^{8}$. 
A large number of in vitro protocols have been established to generate tolDCs that exert prophylactic impacts on Th1-mediated immunopathogenesis, whereas how Th2-mediated allergic reactions are modulated remain obscure $^{9,10}$. IL-10, an anti-inflammatory and immunoregulatory cytokine, was confirmed to be a more potent inducer of tolDCs than vitamin $\mathrm{D}(3)$, dexamethasone, TGF $\beta$, and rapamycin ${ }^{11}$. IL-10 can suppress the expression of MHCII and certain costimulatory molecules on DCs; furthermore, IL-10-induced DCs (DC10) can secrete IL-10, leading to Treg activation ${ }^{12,13}$. Koya et al. proved that DC10 can dramatically attenuate lung allergic responses, with high IL-10 production and low T cell activation ${ }^{14}$. In contrast, the role of DC10 in the Th2-mediated inflammation has been indicated to skew the Th1/Th2 balance to Th2 in vivo via selectively preventing IL-12 synthesis ${ }^{15}$.

While IL-10/TGF 3 -activated DCs can abrogate experimental asthma, several research groups have already demonstrated that LPS, which is most frequently used to stimulate DC maturation in vitro to initiate inflammatory responses, can also cause DCs to produce sufficient IL-10 and exert immunosuppressive effects ${ }^{5,16}$. For example, Zhou et al. revealed that LPS pretreatment modified the phenotype of DCs, thus blocking experimental autoimmune encephalomyelitis (EAE) development by suppressing effector CD4+ T cells ${ }^{5}$. Additionally, Kushwah et al. proved that although LPS-activated DC maturation may lead to DC apoptosis, immature DCs can immediately take up apoptotic DCs and then convert into tolDCs that induce augmented TGF $\beta$ secretion and Foxp3+ Treg differentiation ${ }^{17}$. In contrast, BMDCs were found to upregulate expression of CD80 and CD86 following LPS treatment ${ }^{3}$. Taken together, these findings suggest that LPS might play different roles in modulating phenotypes and functions of DCs under various conditions. Moreover, asthma is a disease with complex immune microenvironment. Whether LPS confers tolerogenic capabilities in BMDCs and the potential mechanisms warrant further investigation.

Herein, we critically evaluated the impact of the intraperitoneal adoptive transfer of LPS-treated BMDCs on airway inflammation and pulmonary memory CD4+ T cells, Tregs, and signal transcription molecules expression in asthmatic models.

\section{Materials and methods}

Mice

6-week-old female C57BL/6 mice were obtained from Silaike Experimental Animal Limited Liability Company (Shanghai, China). All animals were treated in accordance with the National Institutes of Health Guidelines and Regulations.

Isolation and characterization of BMDCs from mice

BMDCs were differentiated from BM cells obtained from femurs and tibiae of C57BL/6 mice. Briefly, BM cells were seeded in RPMI-1640 supplemented with $1 \%$ antibiotics/antimycotics and $10 \%$ heat-inactivated fetal calf serum (FCS) containing $20 \mathrm{ng} / \mathrm{ml}$ granulocyte-macrophage colony-stimulating factor (GM-CSF). The total culture period was 9 days. On day 3, fresh medium was added at the same volume as the preexisting medium. On day 6 , half of the culture medium was replaced with fresh medium. On day 8 , nonadherent DCs were resuspended in complete medium supplemented with GM-CSF alone (to generate immature DCs; DCia) or with $50 \mathrm{ng} / \mathrm{mL}$ IL-10 or $10 \mathrm{ng} / \mathrm{mL}$ LPS (to generate tolerogenic cells; DC10 and DClps) for $24 \mathrm{~h}$. On day 9, cells from each culture were pulsed for $2 \mathrm{~h}$ at 37 with $1 \mu \mathrm{M}$ OVA (Sigma-Aldrich, Grade V; St Louis, MO, USA) and then the cells were harvested. Meanwhile, noninduced BM cells were collected and cultured under the same conditions except that there was no GM-CSF in the culture medium for 9 days.

Harvested BMDCs and BM cells were characterized by evaluating the expression of CD11c, CD80, and MHCII. Cells were washed and stained at 4 for 30 min with APC-Cy7 conjugated anti-CD11c, APCconjugated anti-CD80, and FITC-conjugated anti-MHCII (eBioscience, Inc., San Diego, CA, USA) and were subsequently analyzed by flow cytometry (FACS Canto II; BD Biosciences, San Jose, CA, USA) to determine the positive rate for the expression of labeled antigens. All data were analyzed with Flow Jo software.

Establishment of OVA-induced asthmatic mice and adoptive transfer of diverse BMDCs into asthmatic mice 
To establish an asthma phenotype, 6-week-old female C57BL/6 mice were intraperitoneally sensitized with PBS containing $100 \mu \mathrm{g}$ OVA in $2 \mathrm{mg}$ alum or PBS/alum as a control on days 0 and 7 . Animals were then intranasally challenged with $100 \mu \mathrm{g}$ OVA in PBS or PBS as a control under anaesthetic on days 14-18. For the treatment models, $1 \times 10^{6}$ cells (DCia, DC10 or DClps prepared as described above) were intraperitoneally transferred on days 14-16.

At $24 \mathrm{~h}$ after the last challenge, mice were anesthetized, and bronchoalveolar lavage (BAL) was performed with cold PBS. Subsequently, lungs were obtained for histology and fixed with $4 \%$ paraformaldehyde in PBS. BALF was centrifuged ( $1200 \mathrm{rpm}$ for $10 \mathrm{~min}$, at $\left.4^{\circ} \mathrm{C}\right)$, and cells were obtained to assess the total cell number and differential cell counts. All samples were independently processed and assayed.

Isolation of memory CD4+ T cells from OVA-induced asthmatic mice

We established another asthma phenotype in mice to obtain pulmonary memory CD4+ T cells. The OVA sensitization and challenge protocol was similar to the previous protocol, and BMDCs were adoptively transferred to OVA-sensitized mice for 3 consecutive days. However, the mice were not sacrificed on day 19. Mice were rechallenged with OVA for 5 consecutive days (day 35-39) and sacrificed on day 40 for the detection of memory T cells. We generated single-cell suspensions of lung parenchymal cells by enzymatically digesting tissues, and identified pulmonary memory CD4+ T cells with flow cytometry. The phenotypic analysis of effector memory CD4 $+\mathrm{T}$ cells and central memory CD4+ T cells were performed by evaluating the expression of CD4, CD44, CD62L, and CCR7. A total of $1 \times 10^{6}$ cells were washed and subsequently stained with FITCconjugated anti-CD4, PE-conjugated anti-CD44, APC-conjugated anti-CD62L and PerCP-Cy5.5-conjugated anti-CCR7 (eBioscience). All data were analyzed with Flow Jo software.

Mixed lymphocyte reaction (MLR) of memory CD4+ T cells and BMDCs

Pulmonary memory CD4+ T cells and BMDCs were prepared as described above. BMDCs were pretreated with culture medium containing $200 \mu \mathrm{g} / \mathrm{mL}$ mitomycin $\mathrm{C}$ for $20 \mathrm{~min}$ at $37^{\circ} \mathrm{C}$ (Kyowa Hakko Kogyo, Tokyo, Japan). Pulmonary memory CD4+ T cells were labeled with carboxyfluorescein diacetate succinimidyl ester (CFSE, Invitrogen Ltd., UK) prior to culture to evaluate T cell proliferation. Mitomycin C-treated DCs $\left(1 \times 10^{5} /\right.$ well $)$ and isolated memory CD4 $\mathrm{T}$ cells $\left(4 \times 10^{5} /\right.$ well $)$ were cocultured in 96 -well plates at a ratio of $1: 4$ at $37^{\circ} \mathrm{C}, 5 \% \mathrm{CO}_{2}$ atmosphere for 1,3 or 5 days respectively. The coculture systems were divided into five groups: the control group, the OVA group, the OVA+DCia group, the OVA+DC10 group and the OVA+DClps group, and BMDCs were generated as previously described. After MLR, the proliferation activity of memory CD4+ T cells was evaluated with CFSE on days 1, 3, and 5. On the last day, cells were stimulated with phorbol myristate acetate (PMA; $100 \mathrm{ng} / \mathrm{ml}$, Sigma) and Inomycin ( $5 \mu \mathrm{mol} / \mathrm{L}$, Sigma) overnight, and then cell supernatants were collected to assess IL- 4 and IFN- $\gamma$ secretion by ELISA.

Pulmonary Tregs of OVA-induced asthmatic mice after the adoptive transfer of DClps

As noted, lung tissues of asthmatic mice that were sacrificed on day 19 were immediately collected to determine the number of Tregs in the lung. Single-cell suspensions of lung parenchymal cells were generated by the enzymatic digestion of tissues, and Tregs were characterized as CD4+CD25+Foxp3+ cells by flow cytometry. A total of $1 \times 10^{6}$ cells were washed and stained with FITC-conjugated anti-CD4, PE-conjugated anti-CD25 and APC-conjugated anti-Foxp3 (eBioscience) antidodies, and subsequently analyzed by flow cytometry. All data were analyzed with Flow Jo software.

BMDC tracking in the lung

We labeled transferred DCs with the fluorescent dye PKH26 (Sigma-Aldrich) according to the instructions. Briefly, we used $1 \mathrm{~mL}$ dilution buffer to suspend $2 \times 10^{6} \mathrm{DCs}$ and then mixed the cell suspension with the same amount of labeling solution containing $4 \times 10^{-6} \mathrm{M}$ PKH26 dye in dilution buffer. After 4 min of incubation at room temperature, we added $2 \mathrm{~mL}$ fetal bovine serum (FBS) to terminate the reaction and washed the cells with a control buffer. Finally, $5 \times 10^{6}$ DCs labeled with PKH26 dye were mixed in $1 \mathrm{~mL}$ PLA-CMC solution for the subsequent adoptive transfer. PKH26-labeled DCs were intraperitoneally injected into asthmatic mice $\left(1 \times 10^{6}\right.$ cells/mouse), as described above. After 1, 3 and 7 days, we obtained lung tissues from each 
animal and acquired single-cell suspensions by the enzymatic dispersal of the lungs. Delivered DCs in the lung were assessed via the expression of MHCII and PKH26 as determined by flow cytometry. All data were analyzed with Flow Jo software.

Histopathologic analysis of the lung

The left lung was removed from each mouse and infused with $4 \%$ paraformaldehyde after BAL, embedded in paraffin, sectioned at $5 \mathrm{um}$, and stained with hematoxylin and eosin (H\&E) according to standard procedures for evaluating histopathological changes in bronchial and lung tissues. Right lung specimens were collected and frozen at $-80 \mathrm{deg}$ C for protein analysis by western blotting (WB).

Peribronchial and perivascular inflammation were estimated with a subjective scale of 0-3 based on a scoring system: a score of 0 indicated that there was no detectable inflammation; 1 indicated that most bronchi or vessels were surrounded by a thin layer (one to five cells thick) of inflammatory cells; 2 indicated that most bronchi were surrounded by a thin layer (1-5 cells) of inflammatory cells; 3 indicated that most bronchi or vessels were surrounded by a thick layer $\left(>5\right.$ cells) of inflammatory cells ${ }^{18}$. The average peribronchial and perivascular inflammation scores reflected the total lung inflammation of treated animals. The score is presented as a mean value of each animal.

Cytokine release capacity of BMDCs

BMDCs (DCia, DC10, and DClps) were harvested after 9 days of culture, as described above. Cells from each culture were pulsed for $2 \mathrm{~h}$ at 37 with $1 \mu \mathrm{M}$ OVA. The cell culture supernatant was collected and stored at -80 for subsequent cytokine detection of IL-10, TGF- $\beta$, MCP-3 and IFN- $\gamma$ by ELISA (R\&D Systems).

WB analysis

Expression levels of STAT1, 4, and 6 and their phosphorylated protein levels in lung digests were assessed by WB. Total protein from mouse right lung tissue was extracted by using RIPA Protein Extracted Reagent (Thermo). $40 \mu \mathrm{g}$ lysate samples were fractionated by $10 \%$ sodium dodecyl sulfate (SDS)-PAGE and transferred to polyvinylidene fluoride (PVDF) membranes (Roche, USA). After incubation with blocking buffer containing $5 \%$ skim milk in TBST $(12.5 \mathrm{mM}$ Tris-HCl pH 7.5, $68.5 \mathrm{mM} \mathrm{NaCl}, 0.1 \%$ Tween 20) for $1 \mathrm{~h}$, the membrane was then incubated with primary antibodies overnight, including rabbit anti-STAT1, and rabbit anti-phosphorylated STAT1, rabbit anti-STAT4, rabbit anti-phosphorylated STAT4, rabbit anti-STAT6, rabbit anti-phosphorylated STAT6 antibodies. The anti-mouse GAPDH antibody was used as a loading control. The membrane was washed with TBST and incubated with horseradish-peroxidase-conjugated secondary antibody (Jackson ImmunoResearch) and then developed with and electrochemiluminescence (ECL) substrate solution (Millipore). The membrane was incubated with rabbit anti-murine GAPDH for assessment of internal protein loading control after incubation with stripping buffer for 15 min at 25 . The density of each band was quantified using image analysis software (ImageJ).

Statistical analysis

All data are representative of at least three independent experiments. Each error bar represents the standard deviation (SD). Multigroup comparisons were assessed by either one-way ANOVA with Tukey's post hoc test or by Wilcoxon signed rank tests (FACS analyses) with a commercial software program (GraphPad Prism 8.0, San Diego, CA). $\mathrm{P}<0.05$ was considered statistically significant.

\section{Results}

\section{Phenotypic and functional characteristics of BMDCs primed under different conditions}

We defined the typical phenotypes and characteristics of BMDCs based on two features: the expression of specific costimulatory molecules and the secretion of certain cytokines. As shown in the results, BM cells were triple negative for CD11c, CD80 and MHCII, while BMDCs were triple positive for those markers (Fig. 1B). Interestingly, the expression levels of CD80 and MHCII were lower on DC10 and DClps than DCia (Fig. 2B). 
In addition to surface molecules expression, the secretion of certain inflammatory cytokines (IL-10, TGF- $\beta$, and IFN- $\gamma$ ) is considered essential for characterizing the tolerogenic potential of BMDCs. BMDCs expressed CCR1, CCR2, and CCR3, all of which bind MCP-3 ${ }^{19}$. Thus, we collected BMDC supernatant and examined IL-10, TGF- $\beta$, MCP-3 and IFN- $\gamma$. Both DC10 and DClps secreted high levels of IL-10 and TGF- $\beta$, yet the secretory capacity of these cytokines was markedly lower in DCia. No distinct differences were found in the secretion of MCP-3 or IFN- $\gamma$ among cells. (Fig. 2A).

\section{Adoptive transfer of LPS-treated BMDCs alleviated airway inflammation in OVA-induced asthmatic mice}

We used OVA sensitization/challenge to establish an asthma mouse model (Fig. 3A). In this experiment, BMDCs were generated in vitro and used to treat asthmatic mice, as noted. After OVA stimulation, we found that the lung tissue of asthmatic mice exhibited typical inflammatory changes, which were characterized by airway wall thickening, and inflammatory cell infiltration (Fig. 3B). DClps treatment significantly improved the lung inflammation, reduced the lung injury score (Fig. 3C), as well as dramatically diminished the number of inflammatory cells in BALF (Fig. 3D). The analysis of cellular composition of BALF showed DClps remarkably decreased the number of neutrophils and increased the number of lymphocytes (Fig. $3 \mathrm{D})$, suggesting that the adoptive transfer of DClps may be involved in attenuating lung inflammation in asthmatic mice.

\section{Adoptively transferred LPS-treated BMDCs effectively migrated to the lungs}

The function of DCs is intimately associated with their capacity to migrate to target organs ${ }^{20}$. It was previously demonstrated that the onset of tolerance in DC10-treated asthmatic mice is progressive; thus, airway hyperresponsiveness (AHR) in mice is mildly attenuated at 2 weeks after transfer and vanishes entirely within 3 weeks $^{4}$. To examine when and whether the injected DClps could successfully migrate to the lungs, we established a mouse model of asthma and treated asthma model mice with diverse DCs that were primed under different conditions. We used the fluorescent dye PHK26 as a marker for the trafficking of transferred DCs in vivo in treated mice, and mice were sacrificed on days 1,3 , and 7 . One day after transfer, DClps labeled with PHK26 appeared in the lungs, with a modest proportion of the labeled cells $(2.26 \%$; absolute BMDCs is 396.). In contrast to DCia with $1.08 \%$ (144 cells total), saline control with $0.188 \%$ (35 cells total) $(\mathrm{p}<0.05)$. On day 3, the proportion of all DCs labeled with PHK26 peaked in the lungs, among which DClps increased the most markedly, representing $5.42 \%$ of the labelled BMDCs, compared to $1.52 \%, 0.98 \%$ in DCia and saline group $(\mathrm{p}<0.05)$. However, the number of DClps strikingly decreased on day $7(0.628 \%$, absolute cell number 82). No detectable difference was found among three groups ( $\mathrm{p}>0.05)$.

These data indicate that DClps migrated to the lungs more effectively and gradually accumulated in the lungs within 1 week. Moreover, the decreased percentage of DClps in the lungs was consistent with the alleviation of lung inflammation in asthmatic mice, which further confirmed that DClps may effectively migrate to the lungs and take effect within 1 week.

\section{Adoptive transfer of LPS-treated BMDCs influences the number and function of pulmonary memory CD4+ T cells in OVA-induced asthmatic mice}

It is widely believed that Th1/Th2 ratio imbalances may lead to the pathogenesis of allergic asthma, in which memory $\mathrm{T}$ cells play a crucial role in the capacity of initiating Th2 response under repeated allergen challenges ${ }^{21}$. Memory T cells can be divided into two distinct subsets, central memory $\mathrm{T}$ cells $\left(\mathrm{T}_{\mathrm{CM}}\right.$ cells $)$ and effector memory $\mathrm{T}$ cells ( $\mathrm{T}_{\mathrm{EM}}$ cells), according to their homing characteristics and effector functions ${ }^{22,23}$. In order to assess whether DClps could suppress the Th2 immune recall response in airways of treated mice, we sought to examine the number and function of memory CD4+ T cells in lung tissues. We thus established asthma model mice and treated these mice with diverse DCs, as described above; however, instead of sacrificing the mice on day 19, we rechallenged the animals with OVA from days 35-39 (Fig. 4A).

On day 40, mice were sacrificed and lungs were removed to obtain single-cell suspensions for the isolation 
of memory CD4+ T cells. We confirmed that both DC10 and DClps observably decreased the pulmonary $\mathrm{CD} 4+\mathrm{CD} 44^{\text {high }}$ memory $\mathrm{T}$ cells, whereas the difference in DCia was negligible compared to the OVA group (Fig. 4B). On the other hand, we next identified CD4+ $\mathrm{T}_{\mathrm{CM}}$ cells that were CD62L+CCR7+ double positive and CD4+ $\mathrm{T}_{\mathrm{EM}}$ cells that were CD62L-CCR7- double negative by flow cytometry (Fig. 4B). Surprisingly, we saw no discernible difference in the proportion of $\mathrm{T}_{\mathrm{CM}}$ and $\mathrm{T}_{\mathrm{EM}}$ cells among mice treated with DClps, DC10 or DCia. To gain insight into this phenomenon, we assessed whether the adoptive transfer of DClps affected the proliferation of pulmonary memory CD4+ T cells with an MLR assay. After MLR, the proliferation activity of pulmonary memory CD4+ T cells was determined by CFSE. Consistent with the above results, it was readily apparent the proliferation activity of pulmonary memory CD4+ T cells isolated from mice in the DC10 and DClps groups was obviously suppressed, compared with the DCia and OVA groups, especially on day 3 and day 5 (Fig. 5). Meanwhile, we collected the cell supernatant of MLR to assess IL-4 and IFN- $\gamma$ secretion by memory CD4 $+\mathrm{T}$ cells. We found that compared with cells in the OVA group, both $\mathrm{T}_{\mathrm{EM}}$ and $\mathrm{T}_{\mathrm{CM}}$ cells exhibited a notable decline in the secretion of IL-4 in the DClps and DC10 groups (Fig. 6A), whereas no treatment effect was detectable in the DCia group (Fig. 6B). Interestingly, in the DClps and DC10 groups, the level of IFN- $\gamma$ dramatically reduced in only $\mathrm{T}_{\mathrm{EM}}$ cells but not in $\mathrm{T}_{\mathrm{CM}}$ cells (Fig. $6 \mathrm{~B})$. Taken together, these data indicate that the adoptive transfer of DClps may inhibit the allergen recall response by suppressing memory $\mathrm{CD} 4+\mathrm{T}$ cells. Moreover, the reduction in memory CD4+ $\mathrm{T}$ cells may well reduce the conversion of Th2 cells, which further results in the downregulation of Th2 cytokines and improves the Th1/Th2 imbalance in the immune response.

\section{Adoptive transfer of LPS-treated BMDCs did not alter the number of pulmonary Tregs}

Recent studies have demonstrated that DC10 treatment is associated with the conversion of Th2 effector $\mathrm{T}$ (Teff) cells into CD4+CD25+ Foxp3+ Tregs in the lungs of treated mice, and these Tregs are probably involved in inflammation regulation ${ }^{24}$. Hence, we sought to examine whether DClps treatment increased the number of pulmonary Tregs in a mouse model of asthma. We isolated $\mathrm{T}$ cells from the lungs of asthmatic mice on day 19 and evaluated their expression of certain markers (CD4, CD25, and Foxp3) by FACS. Furthermore, in line with previous studies that the induction of tolerance did not change the number of CD4+CD25+Foxp3+ cells in the lungs in asthma models but rather resulted in stronger Tregs activity, we saw negligible variations in the number of Tregs in treated mice (Fig. 7B). Therefore, our results suggest that neither DC10 nor DClps treatment altered the number of lung CD4+CD25+Foxp3+ Tregs.

\section{Adoptive transfer of LPS-treated BMDCs decreased the phosphorylation level of STAT6 in OVA-induced asthmatic mice}

Having shown that DClps treatment might be associated with the alleviation of airway inflammation in treated mice, we explored the potential mechanisms for this phenomenon. The STAT1 and STAT4 pathways are considered vital for Th1 differentiation, while the IL-4/IL-13/STAT-6 pathway has been confirmed to be the major modulator of Th2 differentiation in asthma pathophysiology ${ }^{25,26}$. To verify whether DClps modulate the airway inflammation of asthmatic mice by affecting the STATs pathways, we measured the total protein level and phosphorylation levels of STAT1, STAT4, and STAT6 in lung homogenates by WB analysis. We found that in asthmatic mice treated with DClps and DC10, the phosphorylation level of STAT6 protein was remarkably decreased, whereas the phosphorylation level of STAT1 and STAT4 protein merely showed a moderate downward trend that was not statistically significant. We also noted that there were no recognizable changes observed in DCia-treated mice. On the basis of the above findings, we concluded that STAT6 signaling may be involved in the regulation of airway inflammation in asthmatic mice treated with DClps (Fig. 8).

\section{Discussion}

In this study, we generated diverse DCs from the bone marrow of wild-type mice that were stimulated with LPS or IL-10 and delivered these cells to OVA-sensitized/challenged mice. Their impact on pulmonary allergic inflammation, memory CD4+ T cells and Tregs was determined. Apart from the downregulation of costimulatory molecules, the upregulation of IL-10 and TGF- $\beta$ secreted from specific DCs can facilitate 
tolerance ${ }^{10}$. Our results show that DClps were phenotypically similar to previously reported tolDCs ${ }^{27,28}$, which exhibited signally reduced levels of MHCII and CD80 and enhanced levels of inhibitory cytokines (IL-10 and TGF- $\beta$ ) relative to DCia. Our observations further confirm that DCs expressing low levels of costimulatory molecules and high levels of anti-inflammatory cytokines might take on a decided tolerance advantage.

In vivo studies showed intravenous transfer of DC10 reportedly markedly impeded airway allergic inflammation in murine models of OVA-induced asthma ${ }^{14,29}$. Moreover, the intravenous delivery of LPS-induced tolDCs notably prohibited EAE development in a mouse model ${ }^{6}$, and Zheng et al. proved that LPS-activated plasmacytoid DCs effectively alleviated experimental chronic kidney disease ${ }^{30}$. Intriguingly, there is conflicting evidence the intranasal administration of DClps producing high levels of IL-10 enhanced airway inflammation $^{31}$, and the intravenous infusion of DC10 failed to reverse the asthma phenotype in sensitized mice $^{32}$. Whether DClps can be used to ameliorate allergic asthma has not been completely explored. The salient discoveries from our study show for the first time that the intraperitoneal transfer of DClps after OVA challenge considerably reduced inflammatory cell infiltration and the lung injury score in a model of asthma, suggesting that the delivery routes of DClps might influence their protective effect in treated animals. On the other hand, the injection of DCia had negligible regulatory effects on airway inflammation; therefore, our data clearly indicate the pivotal functional differences between LPS-stimulated and nonstimulated DCs in vivo.

When DClps migrate to the lung has not been clarified, nor had it been assessed whether DClps stably occupy the lung in vivo. Huang et al. previously reported that DC10 that were intraperitoneally delivered predominantly migrated to the lungs over 1 week and were visibly reduced within 2 weeks, which corresponded to the time span over which lung inflammation improved ${ }^{13}$. On the other side, DC10 treatment have been shown to induce progressive decreases in Th2 responses (cytokines, eosinophilia, and IgE) to levels near background over the next 2-5 weeks ${ }^{33}$. Others have confirmed that LPS activation is required for migratory activity and antigen presentation by tolDCs generated with dexamethasone ${ }^{34}$. Similarly, our DClps tracking data showed that injected cells well migrated to lungs but were active for only 1 week. It remains unexplored why DClps in this study only affected tolerance over 7 days while others have observed impacts over 2-5 weeks ${ }^{13}, 33$, but the findings were not contradictory since the modelling time of animals and some treatment conditions differed in these studies.

Allergen-specific Th2 memory cells have been viewed as fundamental for the development of allergic asthma in both human and animal models, and DCs regulate the generation of memory $\mathrm{T}$ cells at each stage via the interplay between these cells ${ }^{35}$. We thus rechallenged the animals with OVA and assessed pulmonary memory CD4+ T cells, as described above. Of note, we showed that DClps and DC10, but not DCia, markedly decreased the number and proliferation activity of CD4+CD44 $4^{\text {high }}$ memory T cells. Similarly, our MLR assay data confirm that IL-4 secretion was reduced in both $\mathrm{T}_{\mathrm{CM}}$ and $\mathrm{T}_{\mathrm{EM}}$ cells from DClps-treated mice, but IFN- $\gamma$ was reduced only in $\mathrm{T}_{\mathrm{EM}}$ cells. Nonetheless, there was no difference in the proportion of $\mathrm{T}_{\mathrm{CM}}$ and $\mathrm{T}_{\mathrm{EM}}$ cells in $\mathrm{CD} 4+\mathrm{CD} 44^{\text {high }}$ memory $\mathrm{T}$ cells among the groups. To some extent, this might suppress the Th2-skewing immune response, thereby reducing the airway inflammation in treated mice. In addition, ample experimental evidence supports that $\mathrm{T}_{\mathrm{CM}}$ and $\mathrm{T}_{\mathrm{EM}}$ cells can be converted to the other cell type in many conditions ${ }^{36}$; hence, their exact mutual proportion need to be investigated in the future.

We showed that the number of Tregs in the lungs differed negligibly between the groups, as determined by intracellular staining. Interestingly, these data agree well with previous data showing that DC10 treatment ex vivo and in vivo did not augment the number of CD4+CD25+Foxp3+ T cells but rather contributed to their activation ${ }^{24,33,37}$. It is important to note that Zhou et al. demonstrated that LPS-stimulated DCs cannot modulate Treg-associated molecules on CD4+ T cells such as CD25 and Foxp3 ex vivo ${ }^{38}$. Others have also highlighted that the induction of tolerance does not necessarily lead to elevation in the number of CD4+CD25+ Tregs, but it does unequivocally enhance the expression of IL-10, CTLA4 and LAG-3 in OVAsensitized mice ${ }^{39}$ and house dust mite-sensitized mice ${ }^{12}$, but we did not evaluate these specific markers in our experiments. Nevertheless, there is contradictory evidence showing that regulatory DCs prohibited both 
antigen-specific IgE production and allergic responses via the expansion of CD4+CD25+Foxp3+ Tregs ${ }^{16}$, and Tregs were shown to remarkably increase in kidney-draining lymph nodes and kidneys after the adoptive transfer of DClps ${ }^{30}$; thus, wherein we were unable to unequivocally conclude that DClps have an ignorable impact on the number of Tregs in different diseases.

DCs determine the differentiation of distinct $\mathrm{T}$ cell subsets and complex network of environmental signals and intrinsic cellular mechanisms affect DCs' tolerogenicity. Signal transducer and activator of transcription (STAT) is crucial in cytokines-induced cell responses, for example, IFN- $\gamma$ induced STAT1 phosphorylation and IL-12 induced STAT4 phosphorylation can promote Th1 differentiation, whereas IL-4 induced STAT6 phosphorylation promotes Th2 differentiation. Medoff et al. demonstrated that STAT6 in BMDCs was sufficient for the production of C-C motif chemokine ligand such as CCL17, CCL22, which are critical for Th2 lymphocyte recruitment to allergic airways ${ }^{40}$. In present study, there is a trend of diminished expression of py-STAT4 in DClps group compared to OVA group, but it did not reach statistical significance (p>0.05). Py-STAT6 in DClps and DC10 groups were obviously decreased compared to OVA group, which indicate DClps adoptive transfer reversed OVA-sensitized airway inflammation by inhibiting Th2-mediated inflammation.

In short, BMDCs stimulated with LPS exhibited a tolerogenic phenotype. The intraperitoneal transfer of DClps inhibited the development of Th2 allergic responses by suppressing memory $\mathrm{T}$ cells and decreasing STAT6 phosphorylation in a mouse model of OVA-induced asthma. Further insight into the molecular mechanism and optimal process underlying the DClps-mediated immune tolerance should be investigated in the future.

\section{Author contributions}

Conceived and designed the study: Chen ZH, Jin ML; performed the biological experiments: Min ZH, Zeng YZ, Zhu T; performed the statistical analysis: Zhu T, Cui B, Mao RL; and wrote and modified the paper: Zeng YZ, Jin ML and Chen Z. All authors read and approved the final manuscript.

\section{Acknowledgments}

This work was supported by the National Natural Science Foundation of China (81470211 and 81970023 to Chen Z, 81970060 to Jin M), the Shanghai Health Committee (201840288), the Shanghai Respiratory Research Institute and Yang Scientists Training Program of Zhongshan Hospital, the Shanghai Top-Priority Clinical Key Disciplines Construction Project (2017ZZ02013), and the Shanghai Municipal Key Clinical Specialty (shslczdzk02201).

\section{Conflicts of interest}

The authors declare that they have no conflicts of interest.

\section{References}

1. P G, J V, L Z, C T, S A. Antigen presentation and T cell stimulation by dendritic cells. Annual review of immunology 2002; 20:621-67.

2. Hubo M, Trinschek B, Kryczanowsky F, Tuettenberg A, Steinbrink K, Jonuleit H. Costimulatory molecules on immunogenic versus tolerogenic human dendritic cells. Front Immunol 2013; 4:82.

3. De Sousa C P, CM B, EP S, AM C, RA K. Murine mammary carcinoma cells and CD11c(+) dendritic cells elicit distinct responses to lipopolysaccharide and exhibit differential expression of genes required for TLR4 signaling. Cellular immunology 2010; 266:67-75.

4. M L, W D, X Z, H H, A N, JR G. Therapeutic induction of tolerance by IL-10-differentiated dendritic cells in a mouse model of house dust mite-asthma. Allergy 2011; 66:612-20.

5. F Z, B C, GX Z, A R. Immunotherapy using lipopolysaccharide-stimulated bone marrow-derived dendritic cells to treat experimental autoimmune encephalomyelitis. Clinical and experimental immunology 2014; 
$178: 447-58$.

6. F Z, GX Z, A R. LPS-treated bone marrow-derived dendritic cells induce immune tolerance through modulating differentiation of CD4 regulatory T cell subpopulations mediated by 3G11 and CD127. Immunologic research 2017; 65:630-8.

7. de Aragao-Franca LS, Aragao-Franca LS, Rocha V, Rocha V, Cronemberger-Andrade A, Da CF, et al. Tolerogenic Dendritic Cells Reduce Airway Inflammation in a Model of Dust Mite Triggered Allergic Inflammation. Allergy Asthma Immunol Res 2018; 10:406-19.

8. U S, P R. Tolerogenic dendritic cells: molecular and cellular mechanisms in transplantation. Journal of leukocyte biology 2014; 95:53-69.

9. Bol KF, Schreibelt G, Gerritsen WR, de Vries IJ, Figdor CG. Dendritic Cell-Based Immunotherapy: State of the Art and Beyond. Clin Cancer Res 2016; 22:1897-906.

10. Yoo S, Ha SJ. Generation of Tolerogenic Dendritic Cells and Their Therapeutic Applications. Immune Netw 2016; 16:52-60.

11. MA B, JR K, MS H, JJ Z, van Ham SM, A TB. IL-10-generated tolerogenic dendritic cells are optimal for functional regulatory $\mathrm{T}$ cell induction-a comparative study of human clinical-applicable DC. Clinical immunology (Orlando, Fla.) 2012; 142:332-42.

12. M L, W D, X Z, H H, A N, JR G. Therapeutic induction of tolerance by IL-10-differentiated dendritic cells in a mouse model of house dust mite-asthma. Allergy 2011; 66:612-20.

13. Huang H, Dawicki W, Lu M, Nayyar A, Zhang X, Gordon JR. Regulatory dendritic cell expression of MHCII and IL-10 are jointly requisite for induction of tolerance in a murine model of OVA-asthma. Allergy 2013; 68:1126-35.

14. T K, H M, K T, S M, N M, A B, et al. IL-10-treated dendritic cells decrease airway hyperresponsiveness and airway inflammation in mice. The Journal of allergy and clinical immunology 2007; 119:1241-50.

15. De Smedt T, Van Mechelen M, De Becker G, J U, O L, M M. Effect of interleukin-10 on dendritic cell maturation and function. European journal of immunology 1997; 27:1229-35.

16. S F, N Y, Y I, Y S, K S, K E, et al. Regulatory dendritic cells protect against allergic airway inflammation in a murine asthmatic model. The Journal of allergy and clinical immunology 2008; 121:95-104.

17. R K, J W, JR O, G J, J Z, KA S, et al. Uptake of apoptotic DC converts immature DC into tolerogenic DC that induce differentiation of Foxp3+ Treg. European journal of immunology 2010; 40:1022-35.

18. YG K, CH S, HK Y, PH H, JS K, KS L, et al. Involvement of PTEN in airway hyperresponsiveness and inflammation in bronchial asthma. The Journal of clinical investigation 2003; 111:1083-92.

19. F F, D F, A S, S R, L R, A M, et al. Reduced tumorigenicity and augmented leukocyte infiltration after monocyte chemotactic protein-3 (MCP-3) gene transfer: perivascular accumulation of dendritic cells in peritumoral tissue and neutrophil recruitment within the tumor. Journal of immunology (Baltimore, Md. : 1950) 1998; 161:342-6.

20. J B, RM S. Dendritic cells and the control of immunity. Nature 1998; 392:245-52.

21. AS A, RR H, F A, KA A. Changing survival, memory cell compartment, and T-helper balance of lymphocytes between severe and mild asthma. BMC immunology 2008; 9:73.

22. M P, MK J. Origins of CD4(+) effector and central memory T cells. Nature immunology 2011; 12:467-71.

23. F S, J G, A L. Central memory and effector memory T cell subsets: function, generation, and maintenance. Annual review of immunology 2004; 22:745-63. 
24. H H, W D, X Z, J T, JR G. Tolerogenic dendritic cells induce CD4+CD25hiFoxp3+ regulatory T cell differentiation from CD4+CD25-/loFoxp3- effector T cells. Journal of immunology (Baltimore, Md. : 1950) 2010; 185:5003-10.

25. SS A. Targeting cell signaling in allergic asthma. Signal transduction and targeted therapy 2019; 4:45.

26. Chen Z, Wang S, Erekosima N, Li Y, Hong J, Qi X, et al. IL-4 confers resistance to IL-27-mediated suppression on $\mathrm{CD} 4+\mathrm{T}$ cells by impairing signal transducer and activator of transcription 1 signaling. $\mathrm{J}$ Allergy Clin Immunol 2013; 132:912-21.

27. K S, M W, H J, J K, AH E. Pillars Article: Induction of Tolerance by IL-10-Treated Dendritic Cells. J. Immunol. 1997. 159: 4772-4780. Journal of immunology (Baltimore, Md. : 1950) 2016; 197:1547-55.

28. KK E, S T, F N, KG W, VW Y, QJ P, et al. Early life exposure to lipopolysaccharide suppresses experimental autoimmune encephalomyelitis by promoting tolerogenic dendritic cells and regulatory $\mathrm{T}$ cells. Journal of immunology (Baltimore, Md. : 1950) 2009; 183:298-309.

29. Nayyar A, Dawicki W, Huang H, Lu M, Zhang X, Gordon JR. Induction of prolonged asthma tolerance by IL-10-differentiated dendritic cells: differential impact on airway hyperresponsiveness and the Th2 immunoinflammatory response. J Immunol 2012; 189:72-9.

30. D Z, Q C, VW L, Y W, G Z, Y W, et al. Lipopolysaccharide-pretreated plasmacytoid dendritic cells ameliorate experimental chronic kidney disease. Kidney international 2012; 81:892-902.

31. B A, T F, RD R, AM D, D Q, A H, et al. Lipopolysaccharide stimulation of dendritic cells induces interleukin-10 producing allergen-specific T cells in vitro but fails to prevent allergic airway disease. Experimental lung research 2009; 35:307-23.

32. I B, S S, B K, AB R, J K, J S. Interleukin-10-treated dendritic cells do not inhibit Th2 immune responses in ovalbumin/alum-sensitized mice. International archives of allergy and immunology 2006; 141:61-9.

33. X L, A Y, H H, X Z, J T, B D, et al. Induction of type $2 \mathrm{~T}$ helper cell allergen tolerance by IL-10differentiated regulatory dendritic cells. American journal of respiratory cell and molecular biology 2010; 42:190-9.

34. AE A, DJ S, BL S, RA H, AM P, von Delwig A, et al. LPS activation is required for migratory activity and antigen presentation by tolerogenic dendritic cells. Journal of leukocyte biology 2009; 85:243-50.

35. SJ B, WJ M, CA W, MI G. A role for Th2 T-memory cells in early airway obstruction. Cellular immunology 1996; 170:185-94.

36. JR L, DL F. Generation, persistence and plasticity of CD4 T-cell memories. Immunology 2010; 130:46370.

37. M L, W D, X Z, H H, A N, JR G. Therapeutic induction of tolerance by IL-10-differentiated dendritic cells in a mouse model of house dust mite-asthma. Allergy 2011; 66:612-20.

38. S M, T M, T K, Y N, K K, K T, et al. STAT6 deficiency in a mouse model of allergen-induced airways inflammation abolishes eosinophilia but induces infiltration of CD8+ T cells. Clinical and experimental allergy : journal of the British Society for Allergy and Clinical Immunology 1999; 29:114-23.

39. DH S, PA S, GR Z, LS S, JA T, DJ T, et al. Reversal of airway hyperresponsiveness by induction of airway mucosal CD4+CD25+ regulatory T cells. The Journal of experimental medicine 2006; 203:2649-60.

40. BD M, E S, S H, SY T, BP S, JS D, et al. CD11b+ myeloid cells are the key mediators of Th2 cell homing into the airway in allergic inflammation. Journal of immunology (Baltimore, Md. : 1950) 2009; 182:623-35. 


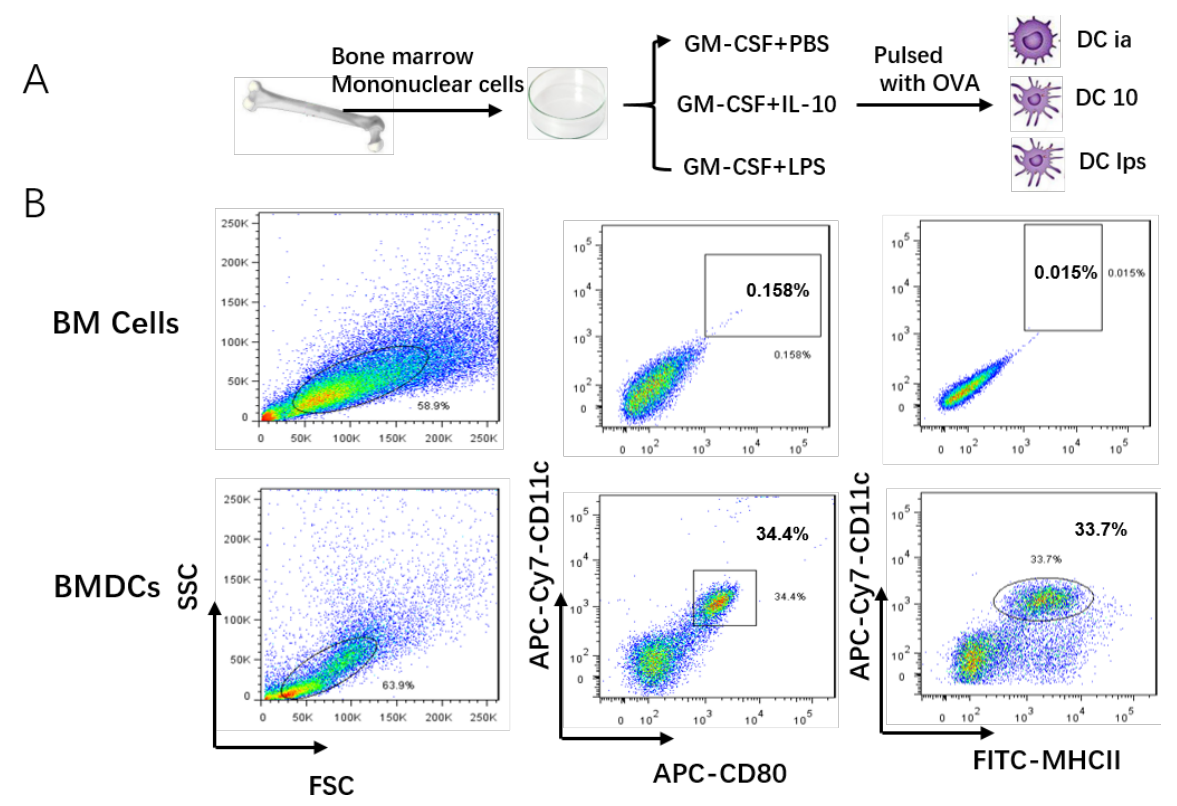

Figure 1 Generation and characterization of BMDCs from mice. BMDCs were generated from mouse femurs and tibiae. BMDCs were cultured in 1640 medium with $10 \%$ FCS and $20 \mathrm{ng} / \mathrm{mL}$ GM-CSF for 9 days. After incubation with PBS, $50 \mathrm{ng} / \mathrm{mL}$ IL-10 or $10 \mathrm{ng} / \mathrm{mL}$ LPS for $24 \mathrm{~h}$, cells were stimulated with $1 \mu \mathrm{M}$ OVA for $2 \mathrm{~h}$, and DCia, DC10 and DClps were generated. CD80, CD11c and MHCII triple -positive cells were identified as BMDCs (lower panel). Noninduced BM cells were triple negative for these markers (upper panel). (A) Schematic of BMDC culture. (B) Characterization of BMDCs by flow cytometry. One representative dot plot is shown.
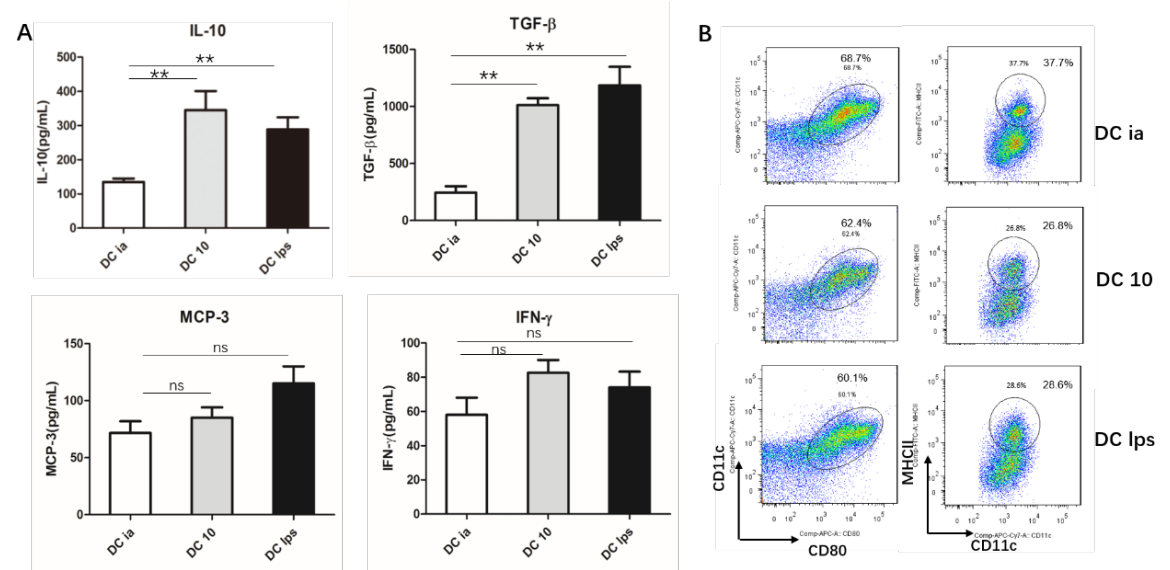

Figure 2 Secretoary capacity and costimulatory molecule expression of BMDCs.

BMDCs incubated with PBS acted as a control. The levels of IL-10, TGF- $\beta$, MCP-3 and IFN- $\gamma$ in the supernatant of DCia, DC10 and DClps culture medium were measured by ELISA. Costimulatory molecules on BMDCs were examined by flow cytometry. CD80 and MHCII expression was lower on DC10 and DClps than DCia. (A) Cytokine and chemokine expression from IL-10- or LPS-activated BMDCs. (B) Costimulatory molecules on BMDCs. Data are representative of 3 independent experiments with similar results. The columns and error bars represent the mean and standard error of the mean (SEM) $(* *$ : $\mathrm{P}<0.01$, ns: no 
significant difference).
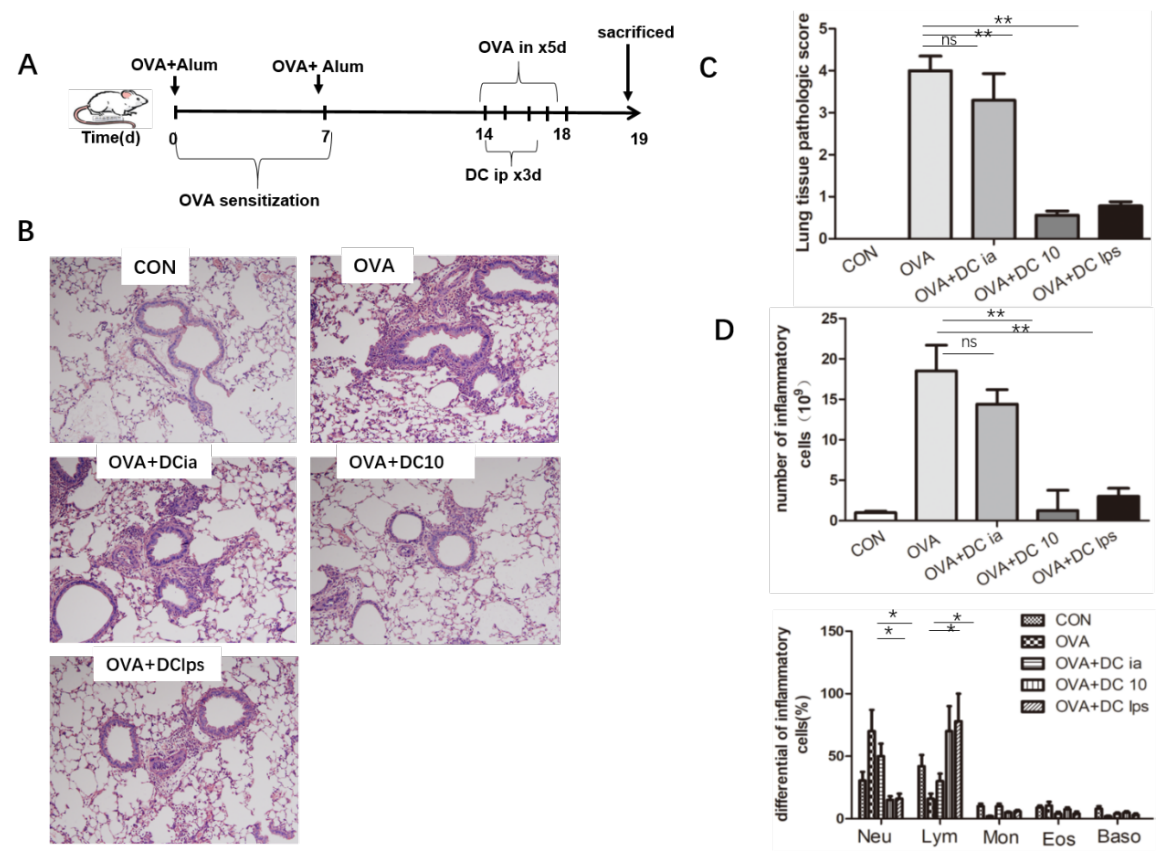

D
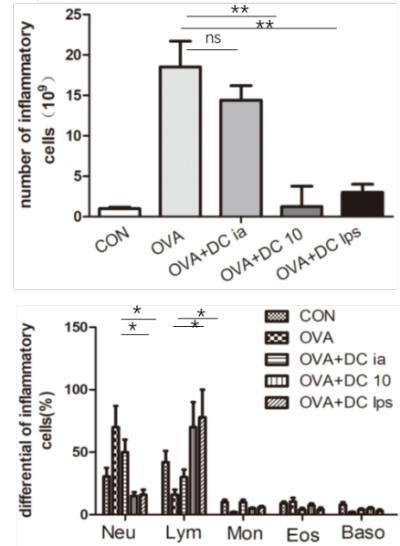

Figure 3 Adoptive transfer of LPS-BMDCs alleviated OVA-induced airway inflammation in asthmatic mice. An asthma model was induced in C57BL/6 mice by OVA-sensitization/challenge. BMDCs were adoptively transferred to OVA-sensitized mice transperitoneally. (A) Schematic of the OVA-induced asthmatic mouse model. (B) Representative H\&E staining of lung sections and images at 100x magnification.

(C) Lung tissue pathology scores. (D) BAL total cell number and differential cell counts. The columns and error bars represent the mean and\& SEM. $\left({ }^{*}: \mathrm{P}<0.05,{ }^{* *}: \mathrm{P}<0.01\right.$, ns: no significant difference). The same experiment was repeated 3 times with similar results $(\mathrm{n}=5$ in each group).
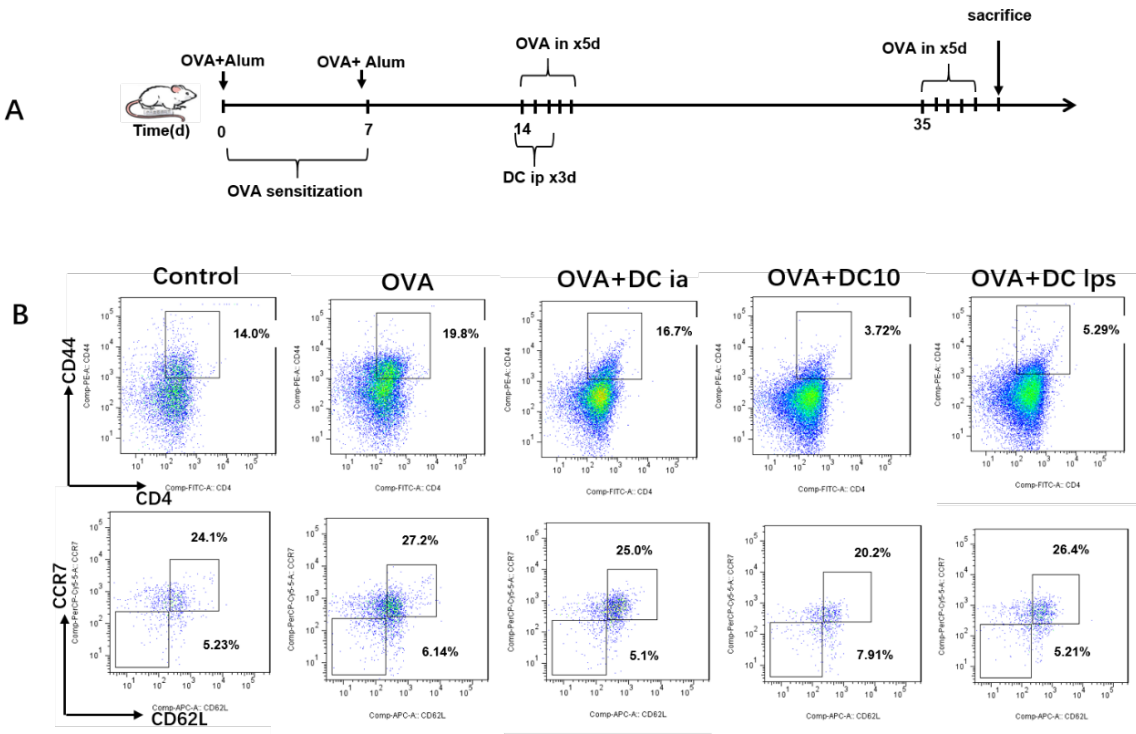
Figure 4 Adoptive transfer of LPS-BMDCs to OVA-induced asthmatic mice reduced the number of pulmonary memory CD4+ T cells. OVA sensitization and challenge was similar to that in the previous schematic. BMDCs were adoptively transferred to OVA-sensitive mice for 3 consecutive days. Rechallenge with OVA was performed for 5 consecutive days starting on day 35. Mice were sacrificed on day 40. Central memory CD4+ T cells were CD62L and CCR7 double positive by flow cytometry. Effector memory CD4+ T cells were CD62L and CCR7 double negative by flow cytometry. The proportion of the two subsets of memory CD4+ T cells was not significantly different among the DCia, DC10 or DClps groups. (A) Schematic of the OVA-induced asthmatic mouse model. (B) Analysis of CD4+CD44high memory T cells by flow cytometry.

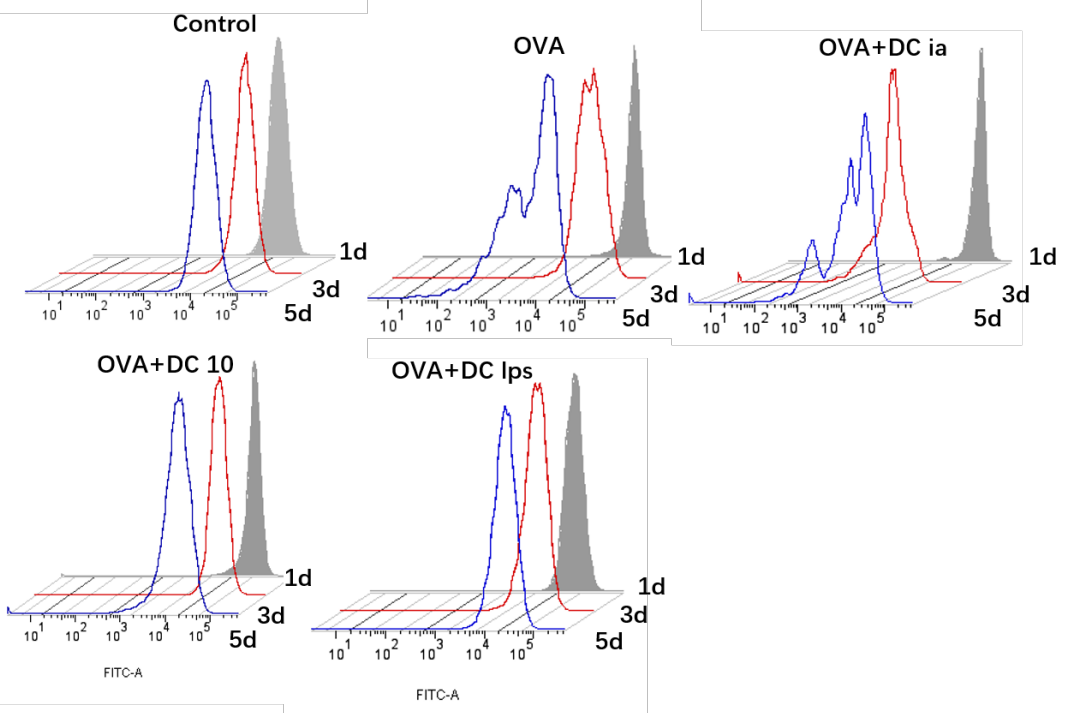

Figure 5 Adoptive transfer of LPS-BMDCs to OVA-sensitized mice inhibits memory CD4+ T cell proliferation in the lung. The schematic of the OVA-induced asthmatic mouse model is similar to that shown in Fig. 4 , and memory CD4+ T cells were obtained from mouse lungs on day 40. After the MLR, the proliferation activity of memory CD4 $+\mathrm{T}$ cells was determined by CFSE. The proliferation of memory CD4+ T cells was lower in the DClps and DC10 groups than the DCia and nontransferred groups. 
A

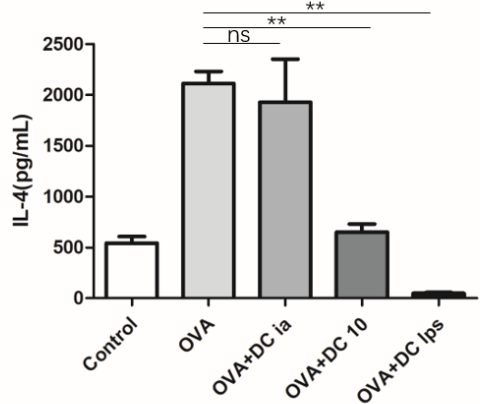

B

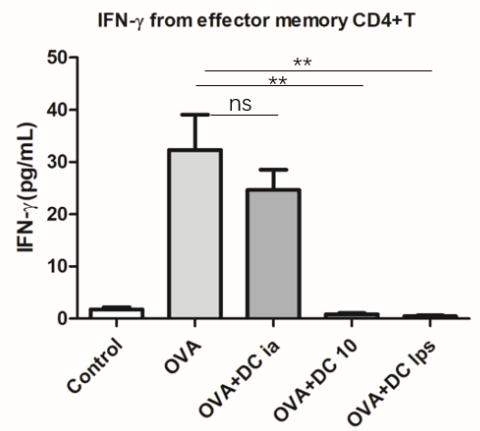

IL-4 from central memory CD4+T

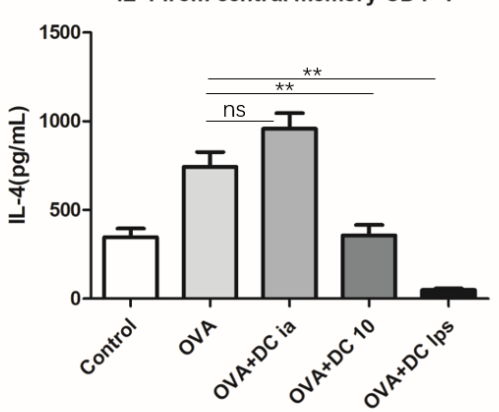

IFN- $\gamma$ from centrol memory CD4+T

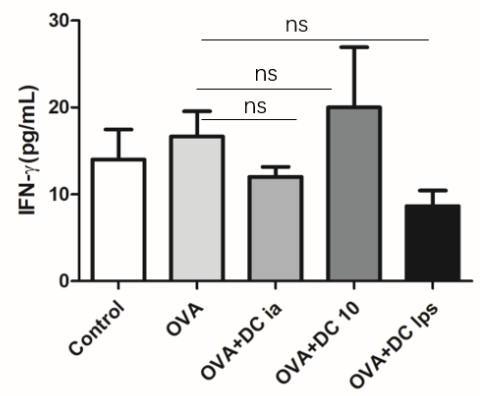

Figure 6 Changes in the cytokine release profile in memory CD4+ T cells from asthmatic mice adoptively transferred with LPS-BMDCs. The schematic of the OVA-induced asthmatic mouse model is similar to that shown in Fig. 4, and memory CD4+ T cells were obtained from mouse lungs on day 40. After MLR, the level of cytokines released from memory CD4 $+\mathrm{T}$ cells was measured by ELISA. IL- 4 secretion was reduced in both effector and central memory CD4 $+\mathrm{T}$ cells from mice that were adoptively transferred DClps and DC10. IFN- $\gamma$ secretion was reduced in effector memory CD4+ T cells but not in central memory CD4+ T cells from mice that were adoptively transferred with DClps and DC10. (A) Th2 cytokine levels. (B) Th1 cytokine levels. The same experiment was repeated 3 times with similar results $(\mathrm{n}=5$ in each group). 


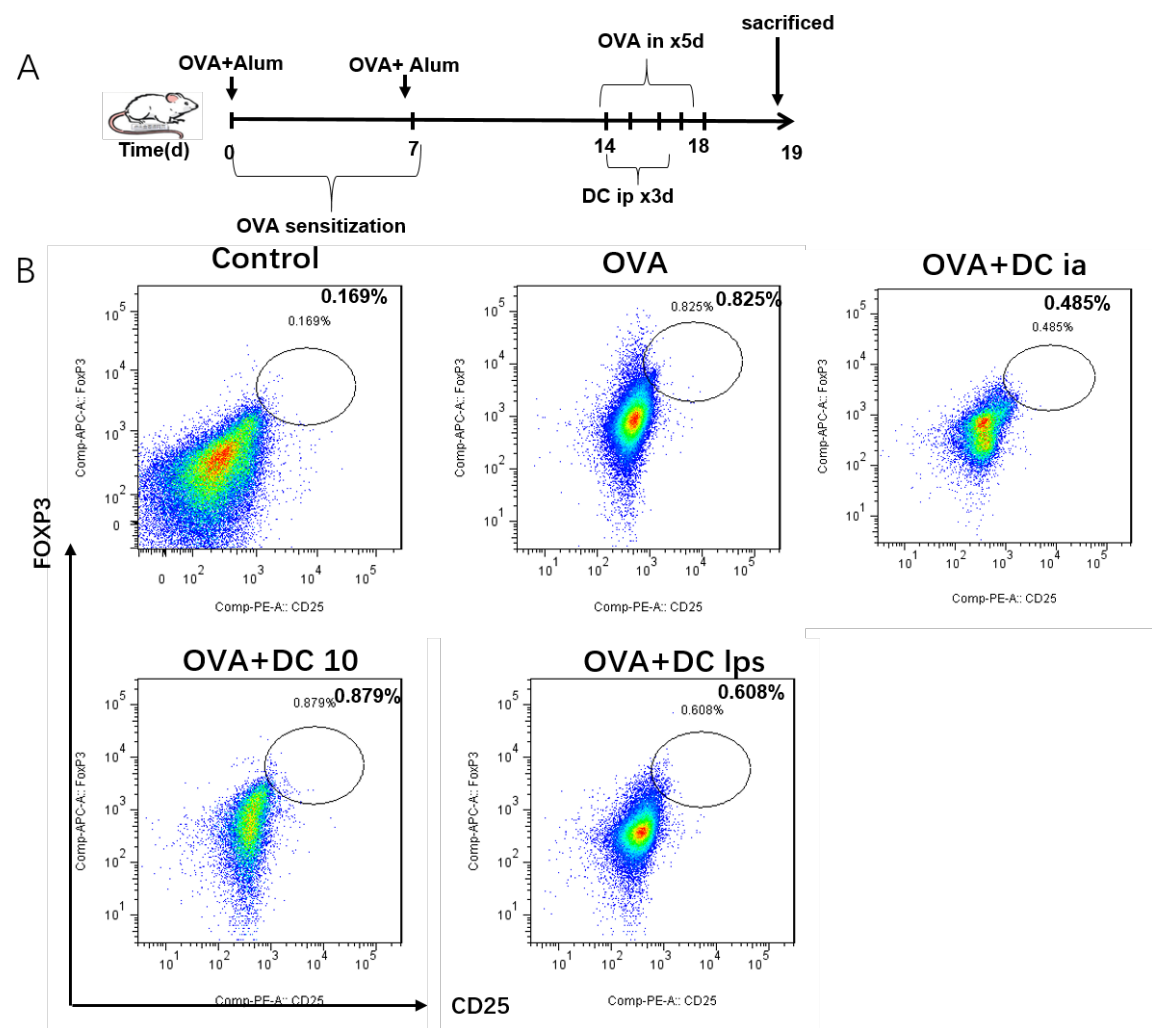

Figure 7 The number of Treg cells in the lung after the transfer of LPS-BMDCs into OVA-induced asthmatic mice. Pulmonary Tregs were characterized as CD4+CD25+Foxp3+ by flow cytometry. The number of Tregs was not significantly different among groups. (A) Schematic of the OVA-induced asthmatic mouse model. (B) Number of pulmonary Tregs.

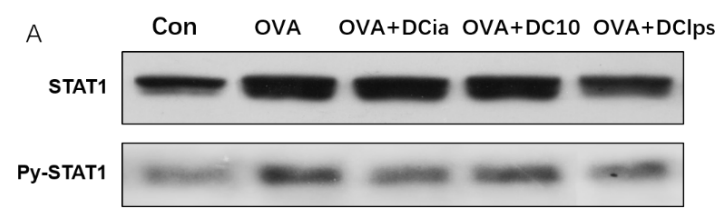

B
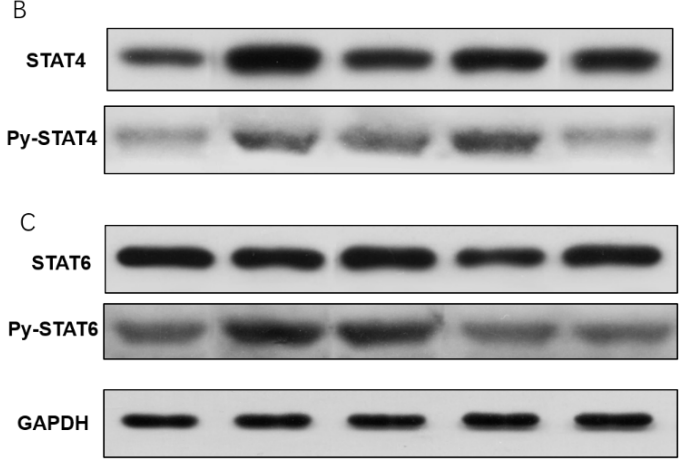
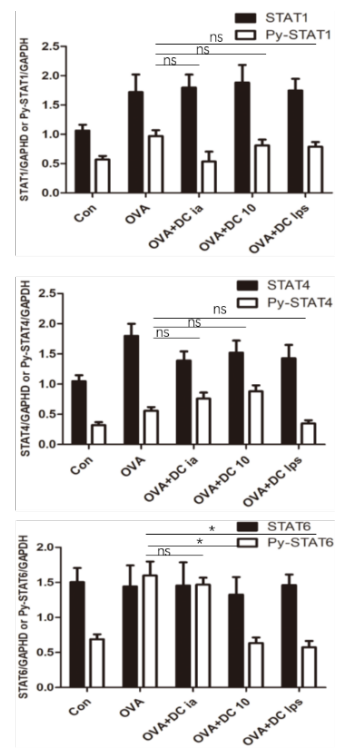

Figure 8 The expression and phosphorylation of STATs in mouse lungs after the transfer of BMDCs to 
OVA-sensitized mice. The protocol for OVA-induced allergic asthma and the transfer of BMDCs to OVAsensitized mice was the same as in Fig. 3.

Lung tissues were homogenized after the adoptive transfer of BMDCs to OVA-sensitized mice. Mononuclear cells were isolated from the homogenate. STATs and phosphorylated STATs were detected by WB. The right columns represent the densitometer analysis of WB. (A) STAT1 and py-STAT1 expression; (B) STAT4 and py-STAT4 expression; (C) STAT6 and py-STAT6 expression. Data are representative of 3 independent experiments with similar results $\left({ }^{*} \mathrm{P}<0.05\right.$, ns: no significant difference). 